\title{
MOTION AND BÄCKLUND TRANSFORMATIONS OF DISCRETE PLANE CURVES
}

\author{
Jun-ichi INOGUCHI, Kenji KAJIWARA, Nozomu MATSUURA and Yasuhiro OHTA
}

(Received 2 March 2011)

\begin{abstract}
We construct explicit solutions to the discrete motion of discrete plane curves that has been introduced by one of the authors recently. Explicit formulas in terms of the $\tau$ function are presented. Transformation theory of the motions of both smooth and discrete curves is developed simultaneously.
\end{abstract}

\section{Introduction}

Differential geometry has a close relationship with the theory of integrable systems. In fact, many integrable differential or difference equations arise as compatibility conditions of some geometric objects. For instance, it is well known that the compatibility condition of pseudospherical surfaces gives rise to the sine-Gordon equation under the Chebyshev net parametrization. For more information on such connections we refer to a monograph [37] by Rogers and Schief.

The above connection between the differential geometry of surfaces and integrable systems has been known since the nineteenth century (although the theory of integrable systems was not yet established). However, it is curious that the link between the differential geometry of curves and integrable systems has only been noticed rather recently. Actually Lamb [28] and Goldstein and Petrich [14] discovered an interesting connection between integrable systems and the differential geometry of plane curves. Namely, they found that the modified Korteweg-de Vries equation ( $\mathrm{mKdV}$ equation) appears as the compatibility condition of a certain motion of plane curves. Here a motion of curves means an isoperimetric time evolution of arc-length parametrized plane curves. More precisely, the compatibility condition implies that the curvature function of a motion should satisfy the mKdV equation. As a result, the angle function of the motion satisfies the potential modified Kortewegde Vries equation (potential $\mathrm{mKdV}$ equation).

On the other hand, in the theory of integrable systems, much attention has been paid to discretization of integrable differential equations preserving integrability, after the pioneering work of Ablowitz and Ladik [1] and Hirota [16-20]. Later, Date, Jimbo and Miwa developed a unified algebraic approach from the viewpoint of the so-called KP theory [5-9, 27, 32]. For other approaches to discrete integrable systems, see, for example, [38, 33]. Thus one can expect the existence of discretized differential geometric objects governed by discrete

2010 Mathematics Subject Classification: Primary 53A04, 37K25, 37K10, 35Q53.

Keywords: discrete curves; discrete motion; discrete potential $\mathrm{mKdV}$ equation; discrete integrable systems; $\tau$ function; Bäcklund transformation.

(C) 2012 Faculty of Mathematics, Kyushu University 
integrable systems. This idea has been realized by the works of Bobenko and Pinkall [3] and Doliwa [10] where the discrete analogue of classical surface theory has been proposed, and it is now actively studied under the name of discrete differential geometry [4].

In the case of the discrete analogue of curves, Doliwa and Santini studied continuous motion of discrete curves in the 3-sphere, and obtained the Ablowitz-Ladik hierarchy in [11], where a semi-discrete (discrete in space variable and continuous in time variable) analogue of the $\mathrm{mKdV}$ equation is derived as the simplest case. Their formulation includes the plane curves as a limiting case. Hisakado et al proposed a discretization of arc-length parametrized plane curves [23], and obtained another semi-discretization of the mKdV equation. Hoffmann and Kutz $[25,26]$ considered discretization of the curvature function. By using their discrete curvature function and Möbius geometry, they obtained a semi-discrete $\mathrm{mKdV}$ equation that is the same as the one in [11].

The discretization of the time variable of discrete curve motion in the 3-sphere was studied in $[\mathbf{1 2}, \mathbf{1 3}]$, where the evolution corresponded to the discrete sine-Gordon equation [18]. As is well known, binormal motion of space curves induces the nonlinear Schrödinger equation via Hasimoto transformation $[\mathbf{1 5}, \mathbf{2 8}]$. Discretization of this curve motion has been formulated in $[\mathbf{2 4}, \mathbf{3 6}]$.

Recently one of the authors of the present paper formulated a full discretization of the motion of plane discrete curves [31] in a purely Euclidean geometric manner, where the discrete potential $\mathrm{mKdV}$ equation proposed by Hirota [21] is deduced as the compatibility condition. It admits a natural continuous limit to the potential $\mathrm{mKdV}$ equation describing continuous motion of smooth plane curves.

In the smooth curve theory, the potential function coincides with the angle function of a curve, a primitive function of the curvature. However, in the discrete case, the potential function and the angle function become different objects. In this framework, the primal geometric object is the potential function rather than the curvature (see $[\mathbf{1 2}, \mathbf{3 1}]$ and Section 2 of the present paper). Natural and systematic construction of the discrete motion of the curves is expected by using the theory of discrete integrable systems.

The purpose of this paper is to construct explicit solutions to discrete motion of discrete plane curves by using the theory of $\tau$ functions. This paper is organized as follows. In Section 2, we prepare fundamental ingredients of plane curve geometry and motions (isoperimetric time evolutions) of plane curves described by the potential $\mathrm{mKdV}$ equation. Next we give a brief review of the discrete motion of discrete curves [31]. In Section 3, we shall give a construction of motions for both smooth and discrete curves by the theory of $\tau$ functions. More precisely we introduce a system of bilinear equations of Hirota type, which can be obtained by a certain reduction of the discrete two-dimensional Toda lattice hierarchy $[\mathbf{2 7}, \mathbf{3 9}, \mathbf{4 0}]$. We shall give a representation formula for curve motions in terms of the $\tau$ function.

One of the central topics in classical differential geometry is the transformation theory of curves and surfaces. The best known example might be the Bäcklund transformations of pseudospherical surfaces. The original Bäcklund transformation was defined as a tangential line congruence satisfying the constant distance property and constant normal angle property (see [37]). In plane curve geometry, Bäcklund transformations on arc-length parametrized plane curves can be defined as arc-length preserving transformations satisfying the constant distance property. Such transformations can be extended to transformations on smooth curve 
motions via the transformation of solutions to the potential $\mathrm{mKdV}$ equation. Motivated by this fact, we shall introduce Bäcklund transformations for discrete motion of discrete curves in Section 4 (compare with [25]). In particular we shall give another type of Bäcklund transformation on motions of both smooth and discrete curves, which is related to the discrete sine-Gordon equation. In Section 5, we shall construct and exhibit some explicit solutions of curve motions, namely, the multi-soliton and multi-breather solutions. We also present some pictures of discrete motions of discrete curves. We finally give some explicit formulas for the Bäcklund transformations of both smooth and discrete curve motions via the $\tau$ functions.

\section{Motion of plane curves}

Let $\gamma(x)$ be an arc-length parametrized curve in the Euclidean plane $\mathbb{R}^{2}$. Then the Frenet equation of $\gamma$ is

$$
\gamma^{\prime \prime}=\left[\begin{array}{cc}
0 & -\kappa \\
\kappa & 0
\end{array}\right] \gamma^{\prime} .
$$

Here ' denotes the differentiation with respect to $x$, and the function $\kappa$ is the curvature of $\gamma$. Let us consider the following motion in time $t$, i.e., isoperimetric time evolution:

$$
\frac{\partial}{\partial t} \gamma^{\prime}=\left[\begin{array}{cc}
0 & \kappa^{\prime \prime}+\frac{\kappa^{3}}{2} \\
-\kappa^{\prime \prime}-\frac{\kappa^{3}}{2} & 0
\end{array}\right] \gamma^{\prime} .
$$

Then the potential function $\theta(x, t)$ defined by $\kappa=\theta^{\prime}$ satisfies the potential $m K d V$ equation $[\mathbf{1 4}, \mathbf{2 8}]$ :

$$
\theta_{t}+\frac{1}{2}\left(\theta_{x}\right)^{3}+\theta_{x x x}=0
$$

The function $\theta$ is called the angle function of $\gamma$ in differential geometry. Note that $\gamma^{\prime}$ can be expressed as

$$
\gamma^{\prime}=\left[\begin{array}{c}
\cos \theta \\
\sin \theta
\end{array}\right] .
$$

For any non-zero constant $\lambda$, the set of equations

$$
\begin{aligned}
& \frac{\partial}{\partial x}\left(\frac{\tilde{\theta}+\theta}{2}\right)=2 \lambda \sin \frac{\tilde{\theta}-\theta}{2}, \\
& \frac{\partial}{\partial t}\left(\frac{\tilde{\theta}+\theta}{2}\right)=-\lambda\left\{\left(\theta_{x}\right)^{2}+8 \lambda^{2}\right\} \sin \frac{\tilde{\theta}-\theta}{2}+2 \lambda \theta_{x x} \cos \frac{\tilde{\theta}-\theta}{2}+4 \lambda^{2} \theta_{x},
\end{aligned}
$$

defines a solution $\widetilde{\theta}$ to the potential $\mathrm{mKdV}$ equation [41]. The solution $\widetilde{\theta}$ is called a Bäcklund transform of $\theta$.

Definition 2.1. A map $\gamma: \mathbb{Z} \rightarrow \mathbb{R}^{2} ; n \mapsto \gamma_{n}$ is said to be a discrete curve of segment length $a_{n}$ if

$$
\left|\frac{\gamma_{n+1}-\gamma_{n}}{a_{n}}\right|=1 .
$$


We introduce the angle function $\Psi_{n}$ of a discrete curve $\gamma$ by

$$
\frac{\gamma_{n+1}-\gamma_{n}}{a_{n}}=\left[\begin{array}{c}
\cos \Psi_{n} \\
\sin \Psi_{n}
\end{array}\right]
$$

A discrete curve $\gamma$ satisfies

$$
\frac{\gamma_{n+1}-\gamma_{n}}{a_{n}}=R\left(K_{n}\right) \frac{\gamma_{n}-\gamma_{n-1}}{a_{n-1}},
$$

for $K_{n}=\Psi_{n}-\Psi_{n-1}$, where $R\left(K_{n}\right)$ denotes the rotation matrix given by

$$
R\left(K_{n}\right)=\left(\begin{array}{cc}
\cos K_{n} & -\sin K_{n} \\
\sin K_{n} & \cos K_{n}
\end{array}\right) .
$$

Now let us recall the following discrete motion of discrete curve $\gamma_{n}^{m}: \mathbb{Z}^{2} \rightarrow \mathbb{R}^{2}$ introduced by Matsuura [31]:

$$
\begin{aligned}
\left|\frac{\gamma_{n+1}^{m}-\gamma_{n}^{m}}{a_{n}}\right| & =1, \\
\frac{\gamma_{n+1}^{m}-\gamma_{n}^{m}}{a_{n}} & =R\left(K_{n}^{m}\right) \frac{\gamma_{n}^{m}-\gamma_{n-1}^{m}}{a_{n-1}}, \\
\frac{\gamma_{n}^{m+1}-\gamma_{n}^{m}}{b_{m}} & =R\left(W_{n}^{m}\right) \frac{\gamma_{n+1}^{m}-\gamma_{n}^{m}}{a_{n}},
\end{aligned}
$$

where $a_{n}$ and $b_{m}$ are arbitrary functions of $n$ and $m$, respectively. Compatibility of the system (2.11)-(2.13) implies the existence of the potential function $\Theta_{n}^{m}$ defined by

$$
W_{n}^{m}=\frac{\Theta_{n}^{m+1}-\Theta_{n+1}^{m}}{2}, \quad K_{n}^{m}=\frac{\Theta_{n+1}^{m}-\Theta_{n-1}^{m}}{2},
$$

and it follows that $\Theta_{n}^{m}$ satisfies the discrete potential $m K d V$ equation [21]:

$$
\tan \left(\frac{\Theta_{n+1}^{m+1}-\Theta_{n}^{m}}{4}\right)=\frac{b_{m}+a_{n}}{b_{m}-a_{n}} \tan \left(\frac{\Theta_{n}^{m+1}-\Theta_{n+1}^{m}}{4}\right) .
$$

Note that the angle function $\Psi_{n}^{m}$ can be expressed as

$$
\Psi_{n}^{m}=\frac{\Theta_{n+1}^{m}+\Theta_{n}^{m}}{2} .
$$

Remark 2.2. The potential discrete mKdV equation (2.15) has also been known as the superposition formula for the modified KdV equation (2.3) [41] and the sine-Gordon equation $[2,37]$.

\section{The $\tau$ function representation of plane curves}

In this section, we give a representation formula for curve motions in terms of $\tau$ functions.

Let $\tau_{n}^{m}=\tau_{n}^{m}(x, t ; y)$ be a complex-valued function dependent on two discrete variables $m$ and $n$, and three continuous variables $x, t$ and $y$, which satisfies the following system of 
bilinear equations:

$$
\begin{aligned}
& \frac{1}{2} D_{x} D_{y} \tau_{n}^{m} \cdot \tau_{n}^{m}=-\left(\tau_{n}^{* m}\right)^{2}, \\
& D_{x}^{2} \tau_{n}^{m} \cdot \tau_{n}^{* m}=0, \\
& \left(D_{x}^{3}+D_{t}\right) \tau_{n}^{m} \cdot \tau_{n}^{* m}=0, \\
& D_{y} \tau_{n+1}^{m} \cdot \tau_{n}^{m}=-a_{n} \tau_{n+1}^{* m} \tau_{n}^{* m}, \\
& D_{y} \tau_{n}^{m+1} \cdot \tau_{n}^{m}=-b_{m} \tau_{n+1}^{* m} \tau_{n}^{* m}, \\
& b_{m} \tau_{n}^{* m+1} \tau_{n+1}^{m}-a_{n} \tau_{n+1}^{* m} \tau_{n}^{m+1}+\left(a_{n}-b_{m}\right) \tau_{n+1}^{* m+1} \tau_{n}^{m}=0 .
\end{aligned}
$$

Here, ${ }^{*}$ denotes the complex conjugate, and $D_{x}, D_{y}$ and $D_{t}$ are Hirota's bilinear differential operators ( $D$-operators) defined by

$$
D_{x}^{i} D_{y}^{j} D_{t}^{k} f \cdot g=\left.\left(\partial_{x}-\partial_{x^{\prime}}\right)^{i}\left(\partial_{y}-\partial_{y^{\prime}}\right)^{j}\left(\partial_{t}-\partial_{t^{\prime}}\right)^{k} f(x, y, t) g\left(x^{\prime}, y^{\prime}, t^{\prime}\right)\right|_{x=x^{\prime}, y=y^{\prime}, t=t^{\prime}} .
$$

For the calculus of the $D$-operators, we refer to [22]. In general, the functions satisfying the bilinear equations of Hirota type are called $\tau$ functions.

THEOREM 3.1. Let $\tau_{n}^{m}$ be a solution to equations (3.1)-(3.6). Define a real function $\Theta_{n}^{m}(x, t ; y)$ and an $\mathbb{R}^{2}$-valued function $\gamma_{n}^{m}(x, t ; y)$ by

$$
\begin{aligned}
\Theta_{n}^{m}(x, t ; y) & :=\frac{2}{\sqrt{-1}} \log \frac{\tau_{n}^{m}}{\tau^{* m}}, \\
\gamma_{n}^{m}(x, t ; y) & :=\left[\begin{array}{c}
-\frac{1}{2}\left(\log \tau_{n}^{m} \tau_{n}^{* m}\right)_{y} \\
\frac{1}{2 \sqrt{-1}}\left(\log \frac{\tau_{n}^{m}}{\tau_{n}^{* m}}\right)_{y}
\end{array}\right] .
\end{aligned}
$$

(1) For any $m, n \in \mathbb{Z}$ and $y \in \mathbb{R}$, the functions $\theta(x, t)=\Theta_{n}^{m}(x, t ; y)$ and $\gamma(x, t)=$ $\gamma_{n}^{m}(x, t ; y)$ satisfy equations $(2.1)-(2.3)$.

(2) For any $x, t, y \in \mathbb{R}$, the functions $\Theta_{n}^{m}=\Theta_{n}^{m}(x, t ; y)$ and $\gamma_{n}^{m}=\gamma_{n}^{m}(x, t ; y)$ satisfy equations (2.11)-(2.15).

Proof. (1) Express $\gamma_{n}^{m}={ }^{t}\left(X_{n}^{m}, Y_{n}^{m}\right)$. Then by using equation (3.1) together with its complex conjugate, we have

$$
\begin{aligned}
\left(X_{n}^{m}\right)^{\prime} & =-\frac{1}{2} \log \left(\tau_{n}^{* m} \tau_{n}^{m}\right)_{x y}=-\frac{1}{2}\left[\frac{\frac{1}{2} D_{x} D_{y} \tau_{n}^{* m} \cdot \tau_{n}^{* m}}{\left(\tau_{n}^{* m}\right)^{2}}+\frac{\frac{1}{2} D_{x} D_{y} \tau_{n}^{m} \cdot \tau_{n}^{m}}{\left(\tau_{n}^{m}\right)^{2}}\right] \\
& =\frac{1}{2}\left[\left(\frac{\tau_{n}^{m}}{\tau_{n}^{* m}}\right)^{2}+\left(\frac{\tau_{n}^{* m}}{\tau_{n}^{m}}\right)^{2}\right]=\cos \Theta_{n}^{m} .
\end{aligned}
$$

Similarly we obtain $\left(Y_{n}^{m}\right)^{\prime}=\sin \Theta_{n}^{m}$. Differentiating $\left(\gamma_{n}^{m}\right)^{\prime}={ }^{\mathrm{t}}\left(\cos \Theta_{n}^{m}, \sin \Theta_{n}^{m}\right)$ by $x$ and noticing that $\kappa=\Theta^{\prime}$, we obtain equation (2.1):

$$
\left(\gamma_{n}^{m}\right)^{\prime \prime}=\left(\Theta_{n}^{m}\right)^{\prime}\left(\begin{array}{c}
-\sin \Theta_{n}^{m} \\
\cos \Theta_{n}^{m}
\end{array}\right)=\left(\begin{array}{cc}
0 & -\kappa \\
\kappa & 0
\end{array}\right)\left(\gamma_{n}^{m}\right)^{\prime} .
$$


On the other hand, differentiating $\left(\gamma_{n}^{m}\right)^{\prime}$ by $t$, we have

$$
\left(\gamma_{n}^{m}\right)_{t}^{\prime}=\left(\Theta_{n}^{m}\right)_{t}\left(\begin{array}{c}
-\sin \Theta_{n}^{m} \\
\cos \Theta_{n}^{m}
\end{array}\right)=\left(\Theta_{n}^{m}\right)_{t}\left(\begin{array}{cc}
0 & -1 \\
1 & 0
\end{array}\right)\left(\gamma_{n}^{m}\right)^{\prime} .
$$

By using the bilinear equations (3.2) and (3.3), $\left(\Theta_{n}^{m}\right)_{t}$ can be rewritten as

$$
\begin{aligned}
\left(\Theta_{n}^{m}\right)_{t} & =\frac{2}{\sqrt{-1}} \frac{D_{t} \tau_{n}^{m} \cdot \tau_{n}^{* m}}{\tau_{n}^{m} \tau_{n}^{* m}}=-\frac{2}{\sqrt{-1}} \frac{D_{x}^{3} \tau_{n}^{m} \cdot \tau_{n}^{* m}}{\tau_{n}^{m} \tau_{n}^{* m}} \\
& =-\frac{2}{\sqrt{-1}}\left[\left(\log \frac{\tau_{n}^{m}}{\tau_{n}^{* m}}\right)_{x x x}+3\left(\log \frac{\tau_{n}^{m}}{\tau_{n}^{* m}}\right)_{x}\left(\log \tau_{n}^{m} \tau_{n}^{* m}\right)_{x x}+\left\{\left(\log \frac{\tau_{n}^{m}}{\tau_{n}^{* m}}\right)_{x}\right\}^{3}\right] \\
& =-\frac{2}{\sqrt{-1}}\left[\left(\log \frac{\tau_{n}^{m}}{\tau_{n}^{* m}}\right)_{x x x}-2\left\{\left(\log \frac{\tau_{n}^{m}}{\tau_{n}^{* m}}\right)_{x}\right\}^{3}\right]=-\kappa_{x x}-\frac{\kappa^{3}}{2}
\end{aligned}
$$

which yields equation (2.2). Here we have used the relation

$$
0=\frac{D_{x}^{2} \tau_{n}^{m} \cdot \tau_{n}^{* m}}{\tau_{n}^{m} \tau_{n}^{* m}}=\left(\log \tau_{n}^{m} \tau_{n}^{* m}\right)_{x x}+\left(\log \frac{\tau_{n}^{m}}{\tau_{n}^{* m}}\right)_{x}^{2},
$$

which is a consequence of equation (3.2). The potential $\mathrm{mKdV}$ equation (2.3) follows immediately from equation (3.10) by noticing that $\kappa=\Theta^{\prime}$.

(2) From equation (3.4) and its complex conjugate we have

$$
\left(\log \frac{\tau_{n+1}^{m}}{\tau_{n}^{m}}\right)_{y}=-a_{n} \frac{\tau_{n+1}^{* m} \tau_{n}^{* m}}{\tau_{n+1}^{m} \tau_{n}^{m}}, \quad\left(\log \frac{\tau_{n+1}^{* m}}{\tau_{n}^{* m}}\right)_{y}=-a_{n} \frac{\tau_{n+1}^{m} \tau_{n}^{m}}{\tau_{n+1}^{* m} \tau_{n}^{* m}} .
$$

Adding these two equations we obtain

$$
\left(\log \tau_{n+1}^{m} \tau_{n+1}^{* m}\right)_{y}-\left(\log \tau_{n}^{m} \tau_{n}^{* m}\right)_{y}=-a_{n}\left(\frac{\tau_{n+1}^{* m} \tau_{n}^{* m}}{\tau_{n+1}^{m} \tau_{n}^{m}}+\frac{\tau_{n+1}^{m} \tau_{n}^{m}}{\tau_{n+1}^{* m} \tau_{n}^{* m}}\right),
$$

which yields

$$
\frac{X_{n+1}^{m}-X_{n}^{m}}{a_{n}}=\cos \Psi_{n}^{m}, \quad \Psi_{n}^{m}=\frac{1}{\sqrt{-1}} \log \left(\frac{\tau_{n+1}^{m} \tau_{n}^{m}}{\tau_{n+1}^{* m} \tau_{n}^{* m}}\right)=\frac{\Theta_{n+1}^{m}+\Theta_{n}^{m}}{2} .
$$

Subtracting the second equation from the first equation in (3.11) we have

$$
\frac{Y_{n+1}^{m}-Y_{n}^{m}}{a_{n}}=\sin \Psi_{n}^{m} .
$$

Therefore we obtain

$$
\frac{\gamma_{n+1}^{m}-\gamma_{n}^{m}}{a_{n}}=\left(\begin{array}{c}
\cos \Psi_{n}^{m} \\
\sin \Psi_{n}^{m}
\end{array}\right),
$$

which gives equation (2.11). Next, from equation (3.14) we see that

$$
\frac{\gamma_{n+1}^{m}-\gamma_{n}^{m}}{a_{n}}=R\left(\Psi_{n}^{m}-\Psi_{n-1}^{m}\right) \frac{\gamma_{n}^{m}-\gamma_{n-1}^{m}}{a_{n-1}}, \quad \Psi_{n}^{m}-\Psi_{n-1}^{m}=\frac{\Theta_{n+1}^{m}-\Theta_{n-1}^{m}}{2}=K_{n}^{m},
$$


which is nothing but equation (2.12). Similarly, starting from equation (3.5) and its complex conjugate we obtain

$$
\frac{\gamma_{n}^{m+1}-\gamma_{n}^{m}}{b_{m}}=\left(\begin{array}{c}
\cos \Phi_{n}^{m} \\
\sin \Phi_{n}^{m}
\end{array}\right), \quad \Phi_{n}^{m}=\frac{1}{\sqrt{-1}} \log \left(\frac{\tau_{n}^{m+1} \tau_{n}^{m}}{\tau_{n}^{* m+1} \tau_{n}^{* m}}\right)=\frac{\Theta_{n}^{m+1}+\Theta_{n}^{m}}{2}
$$

which yields

$$
\frac{\gamma_{n}^{m+1}-\gamma_{n}^{m}}{b_{m}}=R\left(\Phi_{n}^{m}-\Psi_{n}^{m}\right) \frac{\gamma_{n+1}^{m}-\gamma_{n}^{m}}{a_{n}}, \quad \Phi_{n}^{m}-\Psi_{n}^{m}=\frac{\Theta_{n}^{m+1}-\Theta_{n+1}^{m}}{2}=W_{n}^{m} .
$$

This is equivalent to equation (2.13).

Finally let us derive the discrete potential $\mathrm{mKdV}$ equation (2.15). Dividing equation (3.6) and its complex conjugate by $\tau_{n+1}^{* m} \tau_{n}^{* m+1}$ we have

$$
\begin{aligned}
& b_{m} \exp \left(\frac{\sqrt{-1} \Theta_{n+1}^{m}}{2}\right)-a_{n} \exp \left(\frac{\sqrt{-1} \Theta_{n}^{m+1}}{2}\right)=-\left(a_{n}-b_{m}\right) \frac{\tau_{n+1}^{* m+1} \tau_{n}^{m}}{\tau^{* m+1} \tau_{n+1}^{* m}}, \\
& b_{m} \exp \left(\frac{\sqrt{-1} \Theta_{n}^{m+1}}{2}\right)-a_{n} \exp \left(\frac{\sqrt{-1} \Theta_{n+1}^{m}}{2}\right)=-\left(a_{n}-b_{m}\right) \frac{\tau_{n+1}^{m+1} \tau_{n}^{* m}}{\tau_{n}^{* m+1} \tau_{n+1}^{* m}},
\end{aligned}
$$

respectively. Dividing these two equations we obtain

$$
\frac{b_{m} \exp \left(\sqrt{-1} \Theta_{n+1}^{m} / 2\right)-a_{n} \exp \left(\sqrt{-1} \Theta_{n}^{m+1} / 2\right)}{b_{m} \exp \left(\sqrt{-1} \Theta_{n}^{m+1} / 2\right)-a_{n} \exp \left(\sqrt{-1} \Theta_{n+1}^{m} / 2\right)}=\exp \left[-\frac{\sqrt{-1}\left(\Theta_{n+1}^{m+1}-\Theta_{n}^{m}\right)}{2}\right],
$$

which is easily verified to be equivalent to equation (2.15). Thus we have completed the proof of Theorem 3.1.

COROLlARY 3.2. (Representation formula) The function $\gamma_{n}^{m}$ can be expressed in terms of the potential function $\Theta_{n}^{m}$ as follows:

$$
\begin{aligned}
& \gamma_{n}^{m}(x, t ; y)=\left[\begin{array}{l}
\int^{x} \cos \Theta_{n}^{m}\left(x^{\prime}, t ; y\right) d x^{\prime} \\
\int^{x} \sin \Theta_{n}^{m}\left(x^{\prime}, t ; y\right) d x^{\prime}
\end{array}\right] \\
& =\left[\begin{array}{l}
\sum_{n^{\prime}}^{n-1} a_{n^{\prime}} \cos \left(\frac{\Theta_{n^{\prime}}^{m}(x, t ; y)+\Theta_{n^{\prime}+1}^{m}(x, t ; y)}{2}\right) \\
\sum_{n^{\prime}}^{n-1} a_{n^{\prime}} \sin \left(\frac{\Theta_{n^{\prime}}^{m}(x, t ; y)+\Theta_{n^{\prime}+1}^{m}(x, t ; y)}{2}\right)
\end{array}\right] .
\end{aligned}
$$

Proof. The first equation is a consequence of

$$
\frac{\partial}{\partial x} \gamma_{n}^{m}(x, t ; y)=\left[\begin{array}{c}
\cos \Theta_{n}^{m}(x, t ; y) \\
\sin \Theta_{n}^{m}(x, t ; y)
\end{array}\right]
$$

and the second equation follows from equation (3.14). 
It should be noted here that the bilinear equations (3.1)-(3.6) are derived from the reduction of the equations

$$
\begin{aligned}
& \frac{1}{2} D_{x} D_{y} \tau_{n}^{m}(s) \cdot \tau_{n}^{m}(s)=-\tau_{n}^{m}(s+1) \tau_{n}^{m}(s-1), \\
& \left(D_{x}^{2}-D_{z}\right) \tau_{n}^{m}(s+1) \cdot \tau_{n}^{m}(s)=0, \\
& \left(D_{x}^{3}+D_{t}+3 D_{x} D_{z}\right) \tau_{n}^{m}(s+1) \cdot \tau_{n}^{m}(s)=0, \\
& D_{y} \tau_{n+1}^{m}(s) \cdot \tau_{n}^{m}(s)=-a_{n} \tau_{n+1}^{m}(s+1) \tau_{n}^{m}(s-1), \\
& D_{y} \tau_{n}^{m+1}(s) \cdot \tau_{n}^{m}(s)=-b_{m} \tau_{n+1}^{m}(s+1) \tau_{n}^{m}(s-1), \\
& b_{m} \tau_{n}^{m+1}(s+1) \tau_{n+1}^{m}(s)-a_{n} \tau_{n+1}^{m}(s+1) \tau_{n}^{m+1}(s)+\left(a_{n}-b_{m}\right) \tau_{n+1}^{m+1}(s+1) \tau_{n}^{m}(s)=0,
\end{aligned}
$$

for $\tau_{n}^{m}(s)=\tau_{n}^{m}(x, z, t ; y ; s)$, which are included in the discrete two-dimensional Toda lattice hierarchy $[\mathbf{2 7}, \mathbf{3 9}, \mathbf{4 0}]$. In fact, imposing the conditions

$$
\frac{\partial}{\partial z} \tau_{n}^{m}(s)=B \tau_{n}^{m}(s), \quad \tau_{n}^{m}(s+1)=C \tau_{n}^{* m}(s), \quad B, C \in \mathbb{R},
$$

and denoting $\tau_{n}^{m}=\tau_{n}^{m}(0)$, then equations (3.22)-(3.27) yield equations (3.1)-(3.6), respectively.

\section{Bäcklund transformations}

We start with the following fundamental fact on plane curves.

PROPOSITION 4.1. Let $\gamma(x)$ be an arc-length parametrized curve with angle function $\theta(x)$. Take a non-zero constant $\lambda$ and a solution $\widetilde{\theta}(x)$ to

$$
\left(\frac{\tilde{\theta}+\theta}{2}\right)^{\prime}=2 \lambda \sin \frac{\tilde{\theta}-\theta}{2} .
$$

Then

$$
\tilde{\gamma}(x)=\gamma(x)+\frac{1}{\lambda} R\left(\frac{\tilde{\theta}(x)-\theta(x)}{2}\right) \gamma^{\prime}(x)
$$

is an arc-length parametrized curve with angle function $\tilde{\theta}(x)$. In other words, if $\gamma(x)$ is a solution to equation (2.1), then $\widetilde{\gamma}(x)$ is another solution to equation $(2.1)$ with $\widetilde{\kappa}(x)=\widetilde{\theta}^{\prime}(x)$. The curve $\tilde{\gamma}$ is called a Bäcklund transform of $\gamma$.

Proposition 4.1 can be verified easily by direct computation. We next extend the Bäcklund transformation to those of the motion of a curve.

PROPOSITION 4.2. Let $\gamma(x, t)$ be a motion of an arc-length parametrized curve determined by equations (2.2) and (2.3). Take a Bäcklund transform $\widetilde{\theta}(x, t)$ defined by equations (2.5) and (2.6) of $\theta(x, t)$. Then

$$
\tilde{\gamma}(x, t)=\gamma(x, t)+\frac{1}{\lambda} R\left(\frac{\tilde{\theta}(x, t)-\theta(x, t)}{2}\right) \gamma^{\prime}(x, t)
$$

is a motion of an arc-length parametrized curve with the angle function $\tilde{\theta}(x, t)$. 
Proof. By the preceding proposition, $\tilde{\gamma}$ satisfies the isoperimetric condition $\left|\tilde{\gamma}^{\prime}\right|=1$. Computing the $t$-derivative of $\tilde{\gamma}$ by using (2.6), we can show that $\tilde{\gamma}$ satisfies equation (2.2) with $\widetilde{\kappa}=\widetilde{\theta}^{\prime}$.

Now we introduce a Bäcklund transformation of a discrete curve.

PROPOSITION 4.3. Let $\gamma_{n}$ be a discrete curve of segment length $a_{n}$. Let $\Theta_{n}$ be the potential function defined by

$$
\frac{\gamma_{n+1}-\gamma_{n}}{a_{n}}=\left[\begin{array}{c}
\cos \Psi_{n} \\
\sin \Psi_{n}
\end{array}\right], \quad \Psi_{n}=\frac{\Theta_{n+1}+\Theta_{n}}{2}
$$

For a non-zero constant $\lambda$, take a solution $\widetilde{\Theta}_{n}$ to the following equation:

$$
\tan \left(\frac{\widetilde{\Theta}_{n+1}-\Theta_{n}}{4}\right)=\frac{1 / \lambda+a_{n}}{1 / \lambda-a_{n}} \tan \left(\frac{\widetilde{\Theta}_{n}-\Theta_{n+1}}{4}\right) .
$$

Then

$$
\tilde{\gamma}_{n}=\gamma_{n}+\frac{1}{\lambda} R\left(\frac{\widetilde{\Theta}_{n}-\Theta_{n+1}}{2}\right) \frac{\gamma_{n+1}-\gamma_{n}}{a_{n}}
$$

is a discrete curve with the potential function $\widetilde{\Theta}_{n}$.

Proof. It suffices to show that

$$
\frac{\tilde{\gamma}_{n+1}-\tilde{\gamma}_{n}}{a_{n}}=\left[\begin{array}{c}
\cos \tilde{\Psi}_{n} \\
\sin \widetilde{\Psi}_{n}
\end{array}\right], \quad \widetilde{\Psi}_{n}=\frac{\widetilde{\Theta}_{n+1}+\widetilde{\Theta}_{n}}{2}
$$

for $\tilde{\gamma}_{n}$ defined by equation (4.6). This follows from equations (4.4) and (4.5).

We next extend the Bäcklund transformation to those of the motion of a discrete curve. In order to do so, we first present the Bäcklund transformation to the discrete potential $\mathrm{mKdV}$ equation.

LEMMA 4.4. Let $\Theta_{n}^{m}$ be a solution to the discrete potential $m K d V$ equation (2.15). A function $\widetilde{\Theta}_{n}^{m}$ satisfying the following system of equations

$$
\begin{aligned}
\tan \left(\frac{\widetilde{\Theta}_{n+1}^{m}-\Theta_{n}^{m}}{4}\right) & =\frac{1 / \lambda+a_{n}}{1 / \lambda-a_{n}} \tan \left(\frac{\widetilde{\Theta}_{n}^{m}-\Theta_{n+1}^{m}}{4}\right), \\
\tan \left(\frac{\widetilde{\Theta}_{n}^{m+1}-\Theta_{n}^{m}}{4}\right) & =\frac{1 / \lambda+b_{m}}{1 / \lambda-b_{m}} \tan \left(\frac{\widetilde{\Theta}_{n}^{m}-\Theta_{n}^{m+1}}{4}\right),
\end{aligned}
$$

gives another solution to equation (2.15). We call $\widetilde{\Theta}_{n}^{m}$ a Bäcklund transform of $\Theta_{n}^{m}$.

Proof. First note that equation (2.15) is equivalent to

$$
e^{U_{n}^{m+1}+U_{n}^{m}}-e^{U_{n+1}^{m+1}+U_{n+1}^{m}}=\frac{a_{n}}{b_{m}}\left(e^{U_{n+1}^{m}+U_{n}^{m}}-e^{U_{n+1}^{m+1}+U_{n}^{m+1}}\right),
$$

where we put $\sqrt{-1} \Theta_{n}^{m} / 2=U_{n}^{m}$ for notational simplicity. Similarly, equations (4.8) and (4.9) are rewritten as

$$
\begin{gathered}
e^{\widetilde{U}_{n}^{m}+U_{n}^{m}}-e^{\widetilde{U}_{n+1}^{m}+U_{n+1}^{m}}=\lambda a_{n}\left(e^{U_{n+1}^{m}+U_{n}^{m}}-e^{\widetilde{U}_{n+1}^{m}+\widetilde{U}_{n}^{m}}\right), \\
e^{\widetilde{U}_{n}^{m}+U_{n}^{m}}-e^{\widetilde{U}_{n}^{m+1}+U_{n}^{m+1}}=\lambda b_{m}\left(e^{U_{n}^{m+1}+U_{n}^{m}}-e^{\widetilde{U}_{n}^{m+1}+\widetilde{U}_{n}^{m}}\right),
\end{gathered}
$$


respectively, where $\sqrt{-1} \widetilde{\Theta}_{n}^{m} / 2=\widetilde{U}_{n}^{m}$. Subtracting equation (4.12) from equation (4.11), we have

$$
\begin{aligned}
e^{\widetilde{U}_{n}^{m+1}+U_{n}^{m+1}}-e^{\widetilde{U}_{n+1}^{m}+U_{n+1}^{m}}= & \lambda\left(a_{n} e^{U_{n+1}^{m}+U_{n}^{m}}-b_{m} e^{U_{n}^{m+1}+U_{n}^{m}}\right) \\
& -\lambda\left(a_{n} e^{\widetilde{U}_{n+1}^{m}+\widetilde{U}_{n}^{m}}-b_{m} e^{\widetilde{U}_{n}^{m+1}+\widetilde{U}_{n}^{m}}\right) .
\end{aligned}
$$

Similarly, subtracting equation (4.12) $)_{n \rightarrow n+1}$ from equation $(4.11)_{m \rightarrow m+1}$, we get

$$
\begin{aligned}
e^{\widetilde{U}_{n}^{m+1}+U_{n}^{m+1}}-e^{\widetilde{U}_{n+1}^{m}+U_{n+1}^{m}}= & \lambda\left(a_{n} e^{U_{n+1}^{m+1}+U_{n}^{m+1}}-b_{m} e^{U_{n+1}^{m+1}+U_{n+1}^{m}}\right) \\
& -\lambda\left(a_{n} e^{\widetilde{U}_{n+1}^{m+1}+\widetilde{U}_{n}^{m+1}}-b_{m} e^{\widetilde{U}_{n+1}^{m+1}+\widetilde{U}_{n+1}^{m}}\right) .
\end{aligned}
$$

Subtracting equation (4.14) from equation (4.13) yields

$$
\begin{aligned}
& a_{n}\left(e^{\widetilde{U}_{n+1}^{m}+\widetilde{U}_{n}^{m}}-e^{\widetilde{U}_{n+1}^{m+1}+\widetilde{U}_{n}^{m+1}}\right)-b_{m}\left(e^{\widetilde{U}_{n}^{m+1}+\widetilde{U}_{n}^{m}}-e^{\widetilde{U}_{n+1}^{m+1}+\widetilde{U}_{n+1}^{m}}\right) \\
& \quad=a_{n}\left(e^{U_{n+1}^{m}+U_{n}^{m}}-e^{U_{n+1}^{m+1}+U_{n}^{m+1}}\right)-b_{m}\left(e^{U_{n}^{m+1}+U_{n}^{m}}-e^{U_{n+1}^{m+1}+U_{n+1}^{m}}\right) .
\end{aligned}
$$

Now we see that the right-hand side of equation (4.15) vanishes since it is equivalent to equation (4.10). Then the left-hand side gives equation (2.15) for $\widetilde{\Theta}_{n}^{m}$.

Proposition 4.5. Let $\gamma_{n}^{m}$ be a discrete motion of a discrete curve. Take a Bäcklund transform $\widetilde{\Theta}_{n}^{m}$ of $\Theta_{n}^{m}$ defined in Lemma 4.4. Then

$$
\tilde{\gamma}_{n}^{m}=\gamma_{n}^{m}+\frac{1}{\lambda} R\left(\frac{\widetilde{\Theta}_{n}^{m}-\Theta_{n+1}^{m}}{2}\right) \frac{\gamma_{n+1}^{m}-\gamma_{n}^{m}}{a_{n}}
$$

is a discrete motion of a discrete curve with potential function $\widetilde{\Theta}_{n}^{m}$. We call $\widetilde{\gamma}_{n}^{m}$ a Bäcklund transform of $\gamma_{n}^{m}$.

Proof. It suffices to show that $\widetilde{\gamma}_{n}^{m}$ satisfies equations (2.11)-(2.13) with potential function $\widetilde{\Theta}_{n}^{m}$. But equations (2.11) and (2.12) follow from Proposition 4.3 immediately. Noticing the symmetry in $n$ and $m$, similar calculations to those in Proposition 4.3 yield

$$
\frac{\widetilde{\gamma}_{n}^{m+1}-\tilde{\gamma}_{n}^{m}}{b_{m}}=\left[\begin{array}{c}
\cos \left(\frac{\widetilde{\Theta}_{n}^{m+1}+\widetilde{\Theta}_{n}^{m}}{2}\right) \\
\sin \left(\frac{\widetilde{\Theta}_{n}^{m+1}+\widetilde{\Theta}_{n}^{m}}{2}\right)
\end{array}\right]
$$

by using equation (4.9). Comparing equations (4.7) and (4.17) we obtain

$$
\frac{\tilde{\gamma}_{n}^{m+1}-\tilde{\gamma}_{n}^{m}}{b_{m}}=R\left(\frac{\widetilde{\Theta}_{n}^{m+1}-\widetilde{\Theta}_{n+1}^{m}}{2}\right) \frac{\tilde{\gamma}_{n+1}^{m}-\tilde{\gamma}_{n}^{m}}{a_{n}},
$$

which implies equation (2.13).

It is possible to construct another type of Bäcklund transformation for motions of both smooth and discrete curves by using the symmetry of the potential mKdV equation (2.3) and discrete potential $\mathrm{mKdV}$ equation (2.15). In fact, if $\theta(x, t)$ is a solution to equation (2.3), then $-\theta(x, t)$ satisfies the same equation. Combining this symmetry and the Bäcklund transformation defined by equations (2.5) and (2.6), we have the following Bäcklund transformation. 
LeMmA 4.6. Let $\theta(x, t)$ be a solution to the potential $m K d V$ equation (2.3). For any nonzero constant $\lambda$, a function $\bar{\theta}(x, t)$ satisfying the following set of equations

$$
\begin{aligned}
\frac{\partial}{\partial x}\left(\frac{\bar{\theta}-\theta}{2}\right) & =2 \lambda \sin \frac{\bar{\theta}+\theta}{2}, \\
\frac{\partial}{\partial t}\left(\frac{\bar{\theta}-\theta}{2}\right) & =-\lambda\left\{\left(\theta_{x}\right)^{2}+8 \lambda^{2}\right\} \sin \frac{\bar{\theta}+\theta}{2}-2 \lambda \theta_{x x} \cos \frac{\bar{\theta}+\theta}{2}-4 \lambda^{2} \theta_{x},
\end{aligned}
$$

gives another solution to equation (2.3).

Lemma 4.6 immediately yields the following Bäcklund transformation for $\gamma(x, t)$.

PROPOSITION 4.7. Let $\gamma(x, t)$ be a motion of an arc-length parametrized curve determined by equations (2.2) and (2.3). Take a Bäcklund transform $\bar{\theta}(x, t)$ of $\theta(x, t)$ defined in Lemma 4.6. Then

$$
\bar{\gamma}(x, t)=S\left[\gamma(x, t)+\frac{1}{\lambda} R\left(-\frac{\bar{\theta}(x, t)+\theta(x, t)}{2}\right) \gamma^{\prime}(x, t)\right], \quad S=\left[\begin{array}{cc}
1 & 0 \\
0 & -1
\end{array}\right],
$$

is a motion of an arc-length parametrized curve with angle function $\bar{\theta}(x, t)$.

Note that equations (4.19) and (4.20) can be derived from equations (2.5) and (2.6) simply by putting $\tilde{\theta}(x, t)=-\bar{\theta}(x, t)$. Moreover, putting

$$
\hat{\gamma}(x, t):=\gamma(x, t)+\frac{1}{\lambda} R\left(-\frac{\bar{\theta}(x, t)+\theta(x, t)}{2}\right) \gamma^{\prime}(x, t),
$$

and noticing equation (2.4) and Proposition 4.2, we have

$$
\hat{\gamma}^{\prime}(x, t)=\left[\begin{array}{c}
\cos (-\bar{\theta}(x, t)) \\
\sin (-\bar{\theta}(x, t))
\end{array}\right]=\left[\begin{array}{c}
\cos \bar{\theta}(x, t) \\
-\sin \bar{\theta}(x, t)
\end{array}\right],
$$

which implies Proposition 4.7.

Similarly, if $\Theta_{n}^{m}$ is a solution to equation (2.15), then $-\Theta_{n}^{m}$ satisfies the same equation. Therefore Lemma 4.4 and Proposition 4.5 lead to the following Bäcklund transformations.

LEMMA 4.8. Let $\Theta_{n}^{m}$ be a solution to the discrete potential $m K d V$ equation (2.15). A function $\bar{\Theta}_{n}^{m}$ satisfying the following system of equations

$$
\begin{aligned}
\tan \left(\frac{\bar{\Theta}_{n+1}^{m}+\Theta_{n}^{m}}{4}\right) & =\frac{1 / \lambda+a_{n}}{1 / \lambda-a_{n}} \tan \left(\frac{\bar{\Theta}_{n}^{m}+\Theta_{n+1}^{m}}{4}\right), \\
\tan \left(\frac{\bar{\Theta}_{n}^{m+1}+\Theta_{n}^{m}}{4}\right) & =\frac{1 / \lambda+b_{m}}{1 / \lambda-b_{m}} \tan \left(\frac{\bar{\Theta}_{n}^{m}+\Theta_{n}^{m+1}}{4}\right),
\end{aligned}
$$

gives another solution to equation (2.15).

Proposition 4.9. Let $\gamma_{n}^{m}$ be a discrete motion of a discrete curve. Take a Bäcklund transform $\bar{\Theta}_{n}^{m}$ of $\Theta_{n}^{m}$ defined in Lemma 4.8. Then

$$
\bar{\gamma}_{n}^{m}=S\left[\gamma_{n}^{m}+\frac{1}{\lambda} R\left(-\frac{\bar{\Theta}_{n}^{m}+\Theta_{n+1}^{m}}{2}\right) \frac{\gamma_{n+1}^{m}-\gamma_{n}^{m}}{a_{n}}\right], \quad S=\left[\begin{array}{cc}
1 & 0 \\
0 & -1
\end{array}\right],
$$

is a discrete motion of a discrete curve with potential function $\bar{\Theta}_{n}^{m}$. 
Remark 4.10.

(1) It may be interesting to point out that equation (4.23) and equation (4.24) can be rewritten as

$$
\begin{aligned}
\sin \left(\frac{\bar{\Theta}_{n+1}^{m}-\Theta_{n+1}^{m}-\bar{\Theta}_{n}^{m}+\Theta_{n}^{m}}{4}\right) & =\lambda a_{n} \sin \left(\frac{\bar{\Theta}_{n+1}^{m}+\Theta_{n+1}^{m}+\bar{\Theta}_{n}^{m}+\Theta_{n}^{m}}{4}\right), \\
\sin \left(\frac{\bar{\Theta}_{n}^{m+1}-\Theta_{n}^{m+1}-\bar{\Theta}_{n}^{m}+\Theta_{n}^{m}}{4}\right) & =\lambda b_{m} \sin \left(\frac{\bar{\Theta}_{n}^{m+1}+\Theta_{n}^{m+1}+\bar{\Theta}_{n}^{m}+\Theta_{n}^{m}}{4}\right),
\end{aligned}
$$

respectively, which are essentially equivalent to the discrete sine-Gordon equation [18].

(2) The Bäcklund transformations described in Propositions 4.2 and 4.5 satisfy the 'constant distance property', i.e., $|\tilde{\gamma}-\gamma| \equiv 1 / \lambda$ or $\left|\tilde{\gamma}_{n}^{m}-\gamma_{n}^{m}\right| \equiv 1 / \lambda$. These transformations may be regarded as the one-dimensional analogue of the original Bäcklund transformations of the pseudospherical surface [37]. On the other hand, the Bäcklund transformations proposed in Propositions 4.7 and 4.9 are characterized by the property $|\bar{\gamma}-S \gamma|=1 / \lambda$.

\section{Explicit solutions}

\subsection{Solitons and breathers}

For $N \in \mathbb{Z}_{\geq 0}$ we define a function $\tau_{n}^{m}(s)=\tau_{n}^{m}(x, t ; y, z ; s)$ by

$$
\tau_{n}^{m}(s)=\exp \left[-\left(x+\sum_{n^{\prime}}^{n-1} a_{n^{\prime}}+\sum_{m^{\prime}}^{m-1} b_{m^{\prime}}\right) y\right] \operatorname{det}\left(f_{s+j-1}^{(i)}\right)_{i, j=1, \ldots, N},
$$

for $(x, t ; y, z) \in \mathbb{R}^{4}$ and $(m, n, s) \in \mathbb{Z}^{3}$. Here $f_{s}^{(i)}=f_{s}^{(i)}(x, t ; y, z ; m, n)(i=1, \ldots, N)$ satisfies the following linear equations:

$$
\begin{gathered}
\frac{\partial f_{s}^{(i)}}{\partial x}=f_{s+1}^{(i)}, \quad \frac{\partial f_{s}^{(i)}}{\partial z}=f_{s+2}^{(i)}, \quad \frac{\partial f_{s}^{(i)}}{\partial t}=-4 f_{s+3}^{(i)}, \quad \frac{\partial f_{s}^{(i)}}{\partial y}=f_{s-1}^{(i)}, \\
\frac{f_{s}^{(i)}(m, n)-f_{s}^{(i)}(m, n-1)}{a_{n-1}}=f_{s+1}^{(i)}(m, n),
\end{gathered}
$$

For $N=0$, we set $\operatorname{det}\left(f_{s+j-1}^{(i)}\right)_{i, j=1, \ldots, N}=1$. A typical example for $f_{s}^{(i)}$ is given by

$$
\begin{aligned}
& f_{s}^{(i)}=e^{\eta_{i}}+e^{\mu_{i}}, \\
& e^{\eta_{i}}=\alpha_{i} p_{i}^{s} \prod_{n^{\prime}}^{n-1}\left(1-a_{n^{\prime}} p_{i}\right)^{-1} \prod_{m^{\prime}}^{m-1}\left(1-b_{m^{\prime}} p_{i}\right)^{-1} e^{p_{i} x+p_{i}^{2} z-4 p_{i}^{3} t+\left(1 / p_{i}\right) y,} \\
& e^{\mu_{j}}=\beta_{i} q_{i}^{s} \prod_{n^{\prime}}^{n-1}\left(1-a_{n^{\prime}} q_{i}\right)^{-1} \prod_{m^{\prime}}^{m-1}\left(1-b_{m^{\prime}} q_{i}\right)^{-1} e^{q_{i} x+q_{i}^{2} z-4 q_{i}^{3} t+\left(1 / q_{i}\right) y,}
\end{aligned}
$$

where $p_{i}, q_{i}, \alpha_{i}$ and $\beta_{i}$ are arbitrary complex constants. 
We note that $\tau_{n}^{m}$ and $f_{s}^{(i)}$ are functions of continuous variables $x, y, z, t$ and discrete variables $m, n, s$, but we will indicate only the relevant variables according to the context, for notational simplicity. Then it is well known that $\tau_{n}^{m}(s)$ satisfies the bilinear equations (3.22)(3.27) $[\mathbf{2 2}, \mathbf{2 7}, \mathbf{2 9}, \mathbf{3 0}, \mathbf{3 4}, \mathbf{3 5}, \mathbf{4 0}]$. Actually by using the linear relations (5.2) and (5.3), equations (3.22)-(3.27) are reduced to the Plücker relations, which are quadratic identities of determinants.

It is possible to construct the solutions to the bilinear equations (3.1)-(3.6) by imposing the reduction condition (3.28) on $\tau_{n}^{m}(s)$ in equation (5.1). Those conditions are realized by putting restrictions on the parameters of the solutions. As an example, we present the multisoliton and multi-breather solutions.

Proposition 5.1. Consider the $\tau$ function

$$
\begin{aligned}
& \tau_{n}^{m}=\exp \left[-\left(x+\sum_{n^{\prime}}^{n-1} a_{n^{\prime}}+\sum_{m^{\prime}}^{m-1} b_{m^{\prime}}\right) y\right] \operatorname{det}\left(f_{j-1}^{(i)}\right)_{i, j=1, \ldots, N}, \\
& f_{s}^{(i)}=e^{\eta_{i}}+e^{\mu_{i}}, \\
& e^{\eta_{i}}=\alpha_{i} p_{i}^{s} \prod_{n^{\prime}}^{n-1}\left(1-a_{n^{\prime}} p_{i}\right)^{-1} \prod_{m^{\prime}}^{m-1}\left(1-b_{m^{\prime}} p_{i}\right)^{-1} e^{p_{i} x-4 p_{i}^{3} t+\left(1 / p_{i}\right) y}, \\
& e^{\mu_{j}}=\beta_{i}\left(-p_{i}\right)^{s} \prod_{n^{\prime}}^{n-1}\left(1+a_{n^{\prime}} p_{i}\right)^{-1} \prod_{m^{\prime}}^{m-1}\left(1+b_{m^{\prime}} p_{i}\right)^{-1} e^{-p_{i} x+4 p_{i}^{3} t-\left(1 / p_{i}\right) y} .
\end{aligned}
$$

(1) Choosing the parameters as

$$
p_{i}, \alpha_{i} \in \mathbb{R}, \quad \beta_{i} \in \sqrt{-1} \mathbb{R} \quad(i=1, \ldots, N),
$$

then $\tau_{n}^{m}$ satisfies the bilinear equations (3.1)-(3.6). This gives the $N$-soliton solution to equations (2.3) and (2.15).

(2) Taking $N=2 M$, and choosing the parameters as

$$
\begin{aligned}
& p_{i}, \alpha_{i}, \beta_{i} \in \mathbb{C} \quad(i=1, \ldots, 2 M), \quad p_{2 k}=p_{2 k-1}^{*} \quad(k=1, \ldots, M), \\
& \alpha_{2 k}=\alpha_{2 k-1}^{*}, \quad \beta_{2 k}=-\beta_{2 k-1}^{*} \quad(k=1, \ldots, M),
\end{aligned}
$$

then $\tau_{n}^{m}$ satisfies the bilinear equations (3.1)-(3.6). This gives the M-breather solution to equations (2.3) and (2.15).

Proof. It is sufficient to show that the conditions in equation (3.28) are satisfied. We first impose the two-periodicity in $s$, i.e., $\tau_{n}^{m}(s+2)=$ const. $\times \tau_{n}^{m}(s)$. For $\tau_{n}^{m}(s)$ in equation $(5.1)$ with entries given by equations (5.4) and (5.5), putting

$$
q_{i}=-p_{i},
$$

we have

$$
f_{s+2}^{(i)}=p_{i}^{2} f_{s}^{(i)},
$$

which implies that

$$
\tau_{n}^{m}(s+2)=A_{N} \tau_{n}^{m}(s), \quad A_{N}=\prod_{i=1}^{N} p_{i}^{2} .
$$




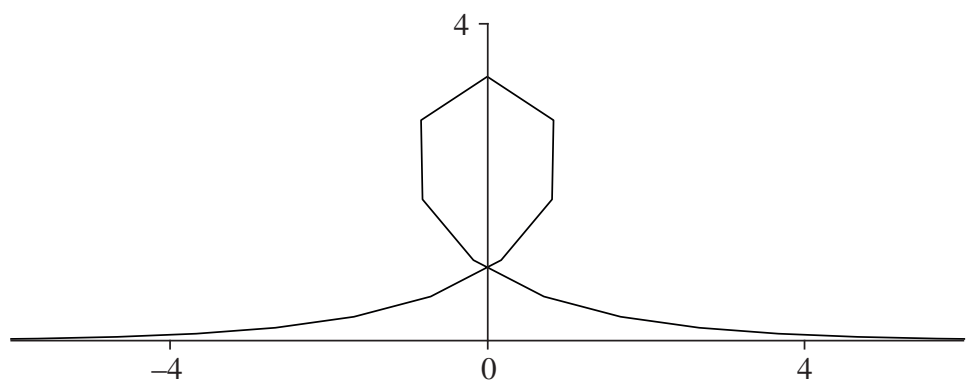

Figure 1. Parameters in equations (5.6), (5.7) and (5.8): $N=1, x=0, y=0, \alpha_{1}=-1, \beta_{1}=\sqrt{-1}$, $p_{1}=0.3, a_{n}=1, b_{m}=0.5$.

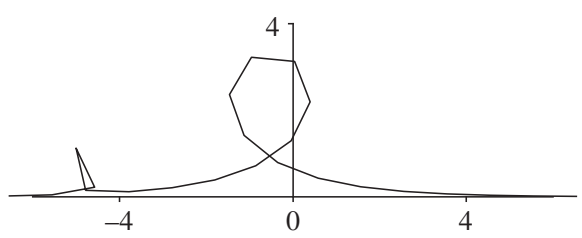

$$
m=-46
$$

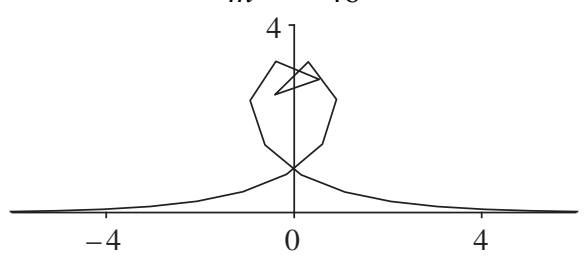

$m=-1$

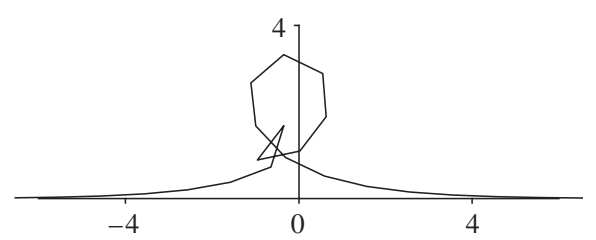

$m=-20$

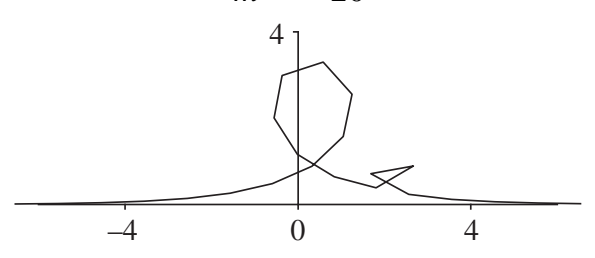

$m=30$

FIGURE 2. Parameters in equations (5.6), (5.7) and (5.8): $N=2, x=0, y=0, \alpha_{1}=-1, \alpha_{2}=1$, $\beta_{1}=-\beta_{2}=\sqrt{-1}, p_{1}=0.3, p_{2}=0.9, a_{n}=1, b_{m}=0.5$.

Note that the condition

$$
\frac{\partial \tau_{n}^{m}(s)}{\partial z}=B_{N} \tau_{n}^{m}(s), \quad B_{N}=\sum_{i=1}^{N} p_{i}^{2},
$$

is also satisfied simultaneously. Now we consider cases (1) and (2) separately.

Case (1). We see from equations (5.7) and (5.8) together with equation (5.9) that

$$
f_{1}^{(i)}=p_{i} f_{0}^{(i) *}
$$

and so

$$
\tau_{n}^{m}(1)=C_{N} \tau_{n}^{* m}(0), \quad C_{N}=\prod_{i=1}^{N} p_{i} \in \mathbb{R} .
$$

Case (2). We see from equations (5.7) and (5.8) together with equation (5.10) that

$$
f_{1}^{(2 k)}=p_{2 k-1}^{*} f_{0}^{(2 k-1) *}, \quad f_{1}^{(2 k-1)}=p_{2 k}^{*} f_{0}^{(2 k) *},
$$



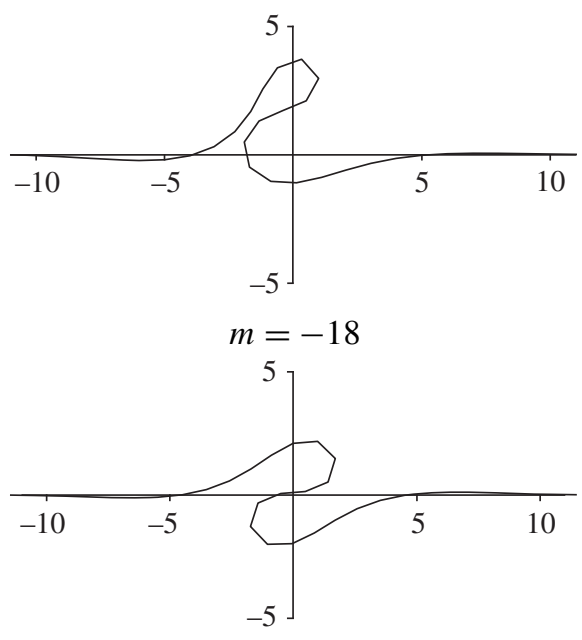

$m=-1$
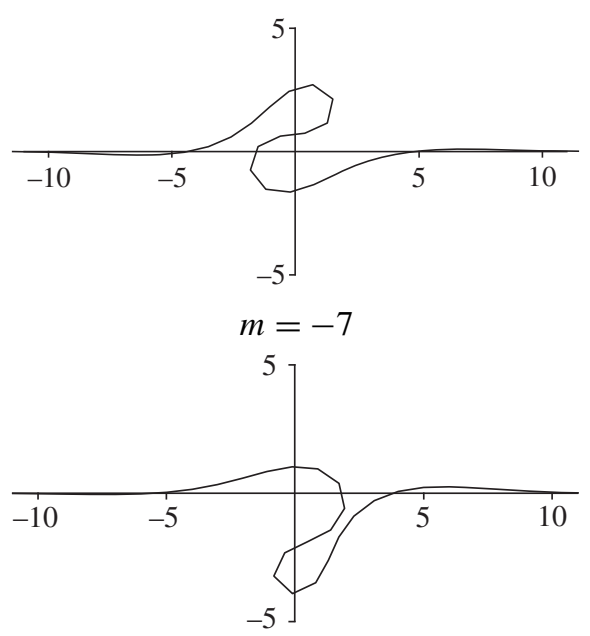

$m=20$

FIGURE 3. Parameters in equations (5.6), (5.7) and (5.8): $N=2, x=0, y=0, \alpha_{1}=\alpha_{2}^{*}=1, \beta_{1}=$ $-\beta_{2}^{*}=1, p_{1}=p_{2}^{*}=0.2-0.2 \sqrt{-1}, a_{n}=1, b_{m}=1.5$.
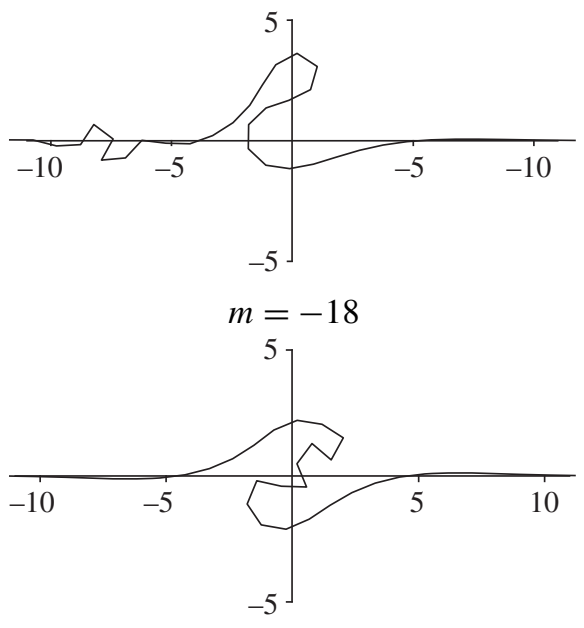

$m=-1$
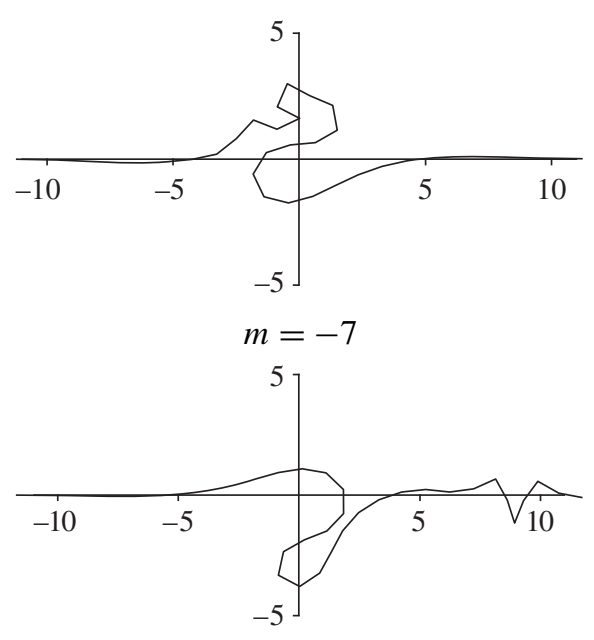

$m=20$

FIGURE 4. Parameters in equations (5.6), (5.7) and (5.8): $N=4, x=0, y=0, \alpha_{1}=\alpha_{2}^{*}=1, \alpha_{3}=$ $\alpha_{4}^{*}=\sqrt{-1}, \beta_{1}=-\beta_{2}^{*}=1, \beta_{3}=-\beta_{4}^{*}=\sqrt{-1}, p_{1}=p_{2}^{*}=0.2-0.2 \sqrt{-1}, p_{3}=p_{4}^{*}=0.8+0.8 \sqrt{-1}$, $a_{n}=1, b_{m}=1.5$. 
and so

$$
\tau_{n}^{m}(1)=C_{N} \tau_{n}^{* m}(0), \quad C_{N}=(-1)^{M} \prod_{i=1}^{M}\left|p_{2 i}\right|^{2} \in \mathbb{R} .
$$

Therefore we have verified that the conditions in equation (3.28) are satisfied for both cases. Then putting $\tau_{n}^{m}=\tau_{n}^{m}(0)$, we obtain the desired result.

We present some pictures of the motions of the discrete curves. Figure 1 shows the simplest example of a curve, which corresponds to the 1-soliton solution (loop soliton). The next example illustrated in Figure 2 describes the interaction of two loops, which corresponds to the 2 -soliton solution. Figures 3 and 4 show the motions which correspond to the 1 -breather and 2-breather solutions, respectively.

\subsection{Solutions via Bäcklund transformations}

In the theory of integrable systems, the Bäcklund transformations are obtained from the shift of a certain discrete independent variable, which also applies to our geometric transformations. We first introduce discrete variables $k, l$, and regard the determinant size $N$ as an additional discrete variable. We then extend the $\tau$ function as $\tau_{n}^{m}(k, l, N)=$ $\tau_{n}^{m}(x, t ; y ; k, l, N)$ in the following way:

$$
\begin{aligned}
& \tau_{n}^{m}(k, l, N)=\exp \left[-\left(x+\sum_{n^{\prime}}^{n-1} a_{n^{\prime}}+\sum_{m^{\prime}}^{m-1} b_{m^{\prime}}+\sum_{k^{\prime}}^{k-1} c_{k^{\prime}}+\sum_{l^{\prime}}^{l-1} \frac{1}{d_{l^{\prime}}}\right) y\right] \operatorname{det}\left(f_{j-1}^{(i)}\right)_{i, j=1, \ldots, N}, \\
& f_{s}^{(i)}=e^{\eta_{i}}+e^{\mu_{i}}, \\
& \left\{\begin{aligned}
e^{\eta_{i}}= & \alpha_{i} p_{i}^{s} \prod_{n^{\prime}}^{n-1}\left(1-a_{n^{\prime}} p_{i}\right)^{-1} \prod_{m^{\prime}}^{m-1}\left(1-b_{m^{\prime}} p_{i}\right)^{-1} \prod_{k^{\prime}}^{k-1}\left(1-c_{k^{\prime}} p_{i}\right)^{-1} \prod_{l^{\prime}}^{l-1}\left(1-\frac{d_{l^{\prime}}}{p_{i}}\right)^{-1} \\
& \times e^{p_{i} x-4 p_{i}^{3} t+\left(1 / p_{i}\right) y}, \\
e^{\mu_{j}}= & \beta_{i}\left(-p_{i}\right)^{s} \prod_{n^{\prime}}^{n-1}\left(1+a_{n^{\prime}} p_{i}\right)^{-1} \prod_{m^{\prime}}^{m-1}\left(1+b_{m^{\prime}} p_{i}\right)^{-1} \prod_{k^{\prime}}^{k-1}\left(1+c_{k^{\prime}} p_{i}\right)^{-1} \prod_{l^{\prime}}^{l-1}\left(1+\frac{d_{l^{\prime}}}{p_{i}}\right)^{-1} \\
& \times e^{-p_{i} x+4 p_{i}^{3} t-\left(1 / p_{i}\right) y} .
\end{aligned}\right.
\end{aligned}
$$

Accordingly, we extend the relevant dependent variables such as $\Theta$ and $\gamma$ in the same way.

PROPOSITION 5.2.

(1) For any $k \in \mathbb{Z}, \tilde{\gamma}(x, t)=\gamma(x, t ; k+1)$ is a Bäcklund transform of $\gamma(x, t)=\gamma(x, t ; k)$ related by equation (4.3) with $\lambda=1 / c_{k}$.

(2) For any $k \in \mathbb{Z}, \widetilde{\gamma}_{n}^{m}=\gamma_{n}^{m}(k+1)$ is a Bäcklund transform of $\gamma_{n}^{m}=\gamma_{n}^{m}(k)$ related by equation (4.16) with $\lambda=1 / c_{k}$.

(3) For any $N \in \mathbb{Z}_{\geq 0}, \tilde{\gamma}(x, t)=\gamma(x, t ; N+1)$ is a Bäcklund transform of $\gamma(x, t)=$ $\gamma(x, t ; N)$ related by equation (4.3) with $\lambda=-p_{N+1}$.

(4) For any $N \in \mathbb{Z}_{\geq 0}, \widetilde{\gamma}_{n}^{m}=\gamma_{n}^{m}(N+1)$ is a Bäcklund transform of $\gamma_{n}^{m}=\gamma_{n}^{m}(N)$ related by equation (4.16) with $\lambda=-p_{N+1}$. 
(5) For any $l \in \mathbb{Z}, \bar{\gamma}(x, t)=\gamma(x, t ; l+1)$ is a Bäcklund transform of $\gamma(x, t)=\gamma(x, t ; l)$ related by equation (4.21) with $\lambda=d_{l}$.

(6) For any $l \in \mathbb{Z}, \bar{\gamma}_{n}^{m}=\gamma_{n}^{m}(l+1)$ is a Bäcklund transform of $\gamma_{n}^{m}=\gamma_{n}^{m}(l)$ related by equation (4.25) with $\lambda=d_{l}$.

Proof. We first prove (1) and (2). It follows from equation (3.4) that the $\tau$ function satisfies the bilinear equation

$$
D_{y} \tau_{n}^{m}(k+1) \cdot \tau_{n}^{m}(k)=-c_{k} \tau_{n}^{* m}(k+1) \tau_{n}^{* m}(k),
$$

because of the symmetry with respect to the discrete variables $m, n$ and $k$ in equations (5.19)(5.21). Then by an argument similar to that in the proof of Theorem 3.1, we see that

$$
\frac{\gamma(k+1)-\gamma(k)}{c_{k}}=\left(\begin{array}{c}
\cos \left(\frac{\theta(k+1)+\theta(k)}{2}\right) \\
\sin \left(\frac{\theta(k+1)+\theta(k)}{2}\right)
\end{array}\right) .
$$

From equation (2.4), we have equation (4.3) with $\tilde{\gamma}=\gamma(k+1)$ and $\widetilde{\theta}=\theta(k+1)$ :

$$
\frac{\gamma(k+1)-\gamma(k)}{c_{k}}=R\left(\frac{\theta(k+1)-\theta(k)}{2}\right) \gamma^{\prime}(k) .
$$

Similarly from equation (3.14), we obtain equation (4.16) with $\widetilde{\gamma}_{n}^{m}=\gamma_{n}^{m}(k+1)$ and $\widetilde{\Theta}_{n}^{m}=$ $\Theta_{n}^{m}(k+1)$ :

$$
\frac{\gamma_{n}^{m}(k+1)-\gamma_{n}^{m}(k)}{c_{k}}=R\left(\frac{\Theta_{n}^{m}(k+1)-\Theta_{n+1}^{m}(k)}{2}\right) \frac{\gamma_{n+1}^{m}(k)-\gamma_{n}^{m}(k)}{a_{n}},
$$

which proves (1) and (2). The statements (3)-(4) and (5)-(6) can be proved in much the same way as (1)-(2), by using the bilinear equations

$$
\begin{aligned}
D_{y} \tau_{n}^{m}(N+1) \cdot \tau_{n}^{m}(N) & =\frac{1}{p_{N+1}} \tau_{n}^{* m}(N) \tau_{n}^{* m}(N+1), \\
D_{y} \tau_{n}^{m}(l+1) \cdot \tau_{n}^{* m}(l) & =-\frac{1}{d_{l}} \tau_{n}^{* m}(l+1) \tau_{n}^{m}(l),
\end{aligned}
$$

respectively. These bilinear equations will be proved in Appendix A.

Remark 5.3. Here we give a physical interpretation of the Bäcklund transformations described above. The Bäcklund transforms in (1)-(2) and (5)-(6) of Propositions 5.2 correspond to changing the phase of solitons (loops), in other words, the positions of solitons. On the other hand, the Bäcklund transforms in (3)-(4) correspond to increasing the number of solitons (loops).

Computing the potential functions of the Bäcklund transforms of the curves, one can verify the following result.

COROLlary 5.4 .

(1) For any $k \in \mathbb{Z}, \tilde{\theta}(x, t)=\theta(x, t ; k+1)$ is a Bäcklund transform of $\theta(x, t)=\theta(x, t ; k)$ related by equations (2.5) and (2.6) with $\lambda=1 / c_{k}$. 
(2) For any $k \in \mathbb{Z}, \widetilde{\Theta}_{n}^{m}=\Theta_{n}^{m}(k+1)$ is a Bäcklund transform of $\Theta_{n}^{m}=\Theta_{n}^{m}(k)$ related by equations (4.8) and (4.9) with $\lambda=1 / c_{k}$.

(3) For any $N \in \mathbb{Z}_{\geq 0}, \tilde{\theta}(x, t)=\theta(x, t ; N+1)$ is a Bäcklund transform of $\theta(x, t)=$ $\theta(x, t ; N)$ related by equations $(2.5)$ and $(2.6)$ with $\lambda=-p_{N+1}$.

(4) For any $N \in \mathbb{Z}_{\geq 0}$, $\widetilde{\Theta}_{n}^{m}=\Theta_{n}^{m}(N+1)$ is a Bäcklund transform of $\Theta_{n}^{m}=\Theta_{n}^{m}(N)$ related by equations (4.8) and (4.9) with $\lambda=-p_{N+1}$.

(5) For any $l \in \mathbb{Z}, \bar{\theta}(x, t)=\theta(x, t ; l+1)$ is a Bäcklund transform of $\theta(x, t)=\theta(x, t ; l)$ related by equations (4.19) and (4.20) with $\lambda=d_{l}$.

(6) For any $l \in \mathbb{Z}, \bar{\Theta}_{n}^{m}=\Theta_{n}^{m}(l+1)$ is a Bäcklund transform of $\Theta_{n}^{m}=\Theta_{n}^{m}(l)$ related by equations (4.23) and (4.24) with $\lambda=d_{l}$.

Acknowledgements. One of the authors (K.K.) would like to thank Professor Tim Hoffmann for giving a series of introductory lectures on discrete differential geometry at Kyushu University. This work is partially supported by JSPS Grant-in-Aid for Scientific Research No. 19340039, 21540067, 21656027 and 22656026.

\section{Appendix A. Derivation of bilinear equations (5.26) and (5.27)}

In this appendix, we show that the $\tau$ function given in equations (5.19)-(5.21) actually satisfies the bilinear equations (5.26) and (5.27). For this purpose, we first introduce the generic $\tau$ function $\tau_{n}^{m}(k, l, N ; s)=\tau_{n}^{m}(x, t ; y, z ; k, l, N ; s)$ by

$$
\begin{aligned}
\tau_{n}^{m}(k, l, N ; s)= & \exp \left[-\left(x+\sum_{n^{\prime}}^{n-1} a_{n^{\prime}}\right.\right. \\
& \left.\left.+\sum_{m^{\prime}}^{m-1} b_{m^{\prime}}+\sum_{k^{\prime}}^{k-1} c_{k^{\prime}}+\sum_{l^{\prime}}^{l-1} \frac{1}{d_{l^{\prime}}}\right) y\right] \operatorname{det}\left(f_{s+j-1}^{(i)}\right)_{i, j=1, \ldots, N},
\end{aligned}
$$

for $(x, t ; y, z) \in \mathbb{R}^{4},(m, n, k, l, s) \in \mathbb{Z}^{5}$ and $N \in \mathbb{Z}_{\geq 0}$. We require $f_{s}^{(i)}=f_{s}^{(i)}(x, t ; y, z$; $m, n ; k, l, N)(i=1, \ldots, N)$ to satisfy the linear equations (5.2), (5.3) and

$$
\frac{f_{s}^{(i)}(k, l)-f_{s}^{(i)}(k-1, l)}{c_{k-1}}=f_{s+1}^{(i)}(k, l), \quad \frac{f_{s}^{(i)}(k, l)-f_{s}^{(i)}(k, l-1)}{d_{l-1}}=f_{s-1}^{(i)}(k, l) .
$$

A typical example for $f_{s}^{(i)}$ is given by

$$
\begin{aligned}
& f_{s}^{(i)}=e^{\eta_{i}}+e^{\mu_{i}}, \\
& e^{\eta_{i}}= \alpha_{i} p_{i}^{s} \prod_{n^{\prime}}^{n-1}\left(1-a_{n^{\prime}} p_{i}\right)^{-1} \prod_{m^{\prime}}^{m-1}\left(1-b_{m^{\prime}} p_{i}\right)^{-1} \prod_{k^{\prime}}^{k-1}\left(1-c_{k^{\prime}} p_{i}\right)^{-1} \prod_{l^{\prime}}^{l-1}\left(1-\frac{d_{l^{\prime}}}{p_{i}}\right)^{-1} \\
& \times e^{p_{i} x-4 p_{i}^{3} t+\left(1 / p_{i}\right) y}, \\
& e^{\mu_{j}}= \beta_{i} q_{i}^{s} \prod_{n^{\prime}}^{n-1}\left(1-a_{n^{\prime}} q_{i}\right)^{-1} \prod_{m^{\prime}}^{m-1}\left(1-b_{m^{\prime}} q_{i}\right)^{-1} \prod_{k^{\prime}}^{k-1}\left(1-c_{k^{\prime}} q_{i}\right)^{-1} \prod_{l^{\prime}}^{l-1}\left(1-\frac{d_{l^{\prime}}}{q_{i}}\right)^{-1} \\
& \times e^{q_{i} x-4 q_{i}^{3} t+\left(1 / q_{i}\right) y},
\end{aligned}
$$


where $p_{i}, q_{i}, \alpha_{i}$ and $\beta_{i}$ are arbitrary complex constants. We put

$$
\sigma_{n}^{m}(y ; k, l, N ; s)=\operatorname{det}\left(f_{s+j-1}^{(i)}\right)_{i, j=1, \ldots, N} .
$$

PROPOSITION A.1. The function $\sigma$ satisfies the following bilinear equations:

$$
\begin{aligned}
& D_{y} \sigma_{n}^{m}(N+1 ; s) \cdot \sigma_{n}^{m}(N ; s)=\sigma_{n}^{m}(N ; s+1) \sigma_{n}^{m}(N+1 ; s-1), \\
& \left(D_{y}-\frac{1}{d_{l}}\right) \sigma_{n}^{m}(l+1 ; s) \cdot \sigma_{n}^{m}(l ; s+1)=-\frac{1}{d_{l}} \sigma_{n}^{m}(l+1 ; s+1) \sigma_{n}^{m}(l ; s) .
\end{aligned}
$$

We apply the determinantal technique in order to prove Proposition A.1. The bilinear equations are reduced to the Plücker relations, which are quadratic identities of determinants whose columns are appropriately shifted. To this end, we construct such formulas that express the determinants in the Plücker relations in terms of the derivative or shift of a discrete variable of $\sigma_{n}^{m}(k, l, N ; s)$ by using the linear relations of the entries. For the details of the technique, we refer to $[\mathbf{2 2}, \mathbf{2 9}, \mathbf{3 0}, \mathbf{3 4}, \mathbf{3 5}]$.

We introduce the notation

$$
\sigma_{n}^{m}(l, N ; s)=\left|0_{l}, 1_{l}, \ldots, N-2_{l}, N-1_{l}\right|,
$$

where ' $j_{l}$ ' denotes the column vector

$$
j_{l}=\left[\begin{array}{c}
f_{s+j}^{(1)}(l) \\
\vdots \\
f_{s+j}^{(N)}(l)
\end{array}\right] .
$$

LEMMA A.2. The following formulas hold:

$$
\begin{aligned}
\partial_{y} \sigma_{n}^{m}(l, N ; s) & =|-1,1, \ldots, N-2, N-1|, \\
\sigma_{n}^{m}(l+1, N ; s) & =\left|0_{l+1}, 1, \ldots, N-2, N-1\right|, \\
d_{l} \sigma_{n}^{m}(l+1, N ; s) & =\left|1_{l+1}, 1, \ldots, N-2, N-1\right|, \\
-\left(d_{l} \partial_{y}-1\right) \sigma_{n}^{m}(l+1, N ; s) & =\left|0,1_{l+1}, 2, \ldots, N-2, N-1\right| .
\end{aligned}
$$

Note that the subscripts of column vectors are shown only when $l$ is shifted for notational simplicity.

Proof. Equation (A.10) can be verified by direct calculation by using the fourth equation in (5.2). We have

$$
\sigma_{n}^{m}(l+1, N ; s)=\left|0_{l+1}, 1_{l+1}, \ldots, N-2_{l+1}, N-1_{l+1}\right| .
$$

Adding the $(N-1)$ th column multiplied by $d_{l}$ to the $N$ th column and using equation (A.2), we have

$$
\sigma_{n}^{m}(l+1, N ; s)=\left|0_{l+1}, 1_{l+1}, \ldots, N-2_{l+1}, N-1_{l}\right| .
$$

Similarly, adding the $(i-1)$ th column multiplied by $d_{l}$ to the $i$ th column and using equation (A.2) for $i=N-1, \ldots, 2$, we obtain

$$
\sigma_{n}^{m}(l+1, N ; s)=\left|0_{l+1}, 1, \ldots, N-2, N-1\right|,
$$


which is equation (A.11). Multiplying $d_{l}$ to the first column of equation (A.11) and using equation (A.2), we obtain equation (A.12). Finally, differentiating equation (A.12) with respect to $y$ yields

$$
\begin{aligned}
& d_{l} \partial_{y} \sigma_{n}^{m}(l+1, N ; s) \\
& \quad=\left|0_{l+1}, 1,2, \ldots, N-2, N-1\right|+\left|1_{l+1}, 0,2, \ldots, N-2, N-1\right| \\
& \quad=\sigma_{n}^{m}(l+1, N ; s)-\left|0,1_{l+1}, 2, \ldots, N-2, N-1\right|,
\end{aligned}
$$

which is equivalent to equation (A.13). This completes the proof.

Proof of Proposition A.1. Consider the Plücker relation (see, for example, [35]),

$$
\begin{aligned}
0= & |-1,0,1, \ldots, N-2| \times|1, \ldots, N-2, N-1, \phi| \\
& +|0,1, \ldots, N-2, N-1| \times|-1,1, \ldots, N-2, \phi| \\
& -|0,1, \ldots, N-2, \phi| \times|-1,1, \ldots, N-2, N-1|,
\end{aligned}
$$

where $\phi$ is a column vector given by

$$
\phi=\left[\begin{array}{c}
0 \\
\vdots \\
0 \\
1
\end{array}\right] .
$$

By using equations (A.8) and (A.10), expanding the determinant with respect to the column $\phi$, equation (A.18) can be rewritten as

$$
\begin{aligned}
0= & \sigma_{n}^{m}(N ; s-1) \sigma_{n}^{m}(N-1 ; s+1)+\sigma_{n}^{m}(N ; s) \partial_{y} \sigma_{n}^{m}(N-1 ; s) \\
& -\sigma_{n}^{m}(N-1 ; s) \partial_{y} \sigma_{n}^{m}(N ; s),
\end{aligned}
$$

which implies equation (A.6). Similarly, applying Lemma A.2 on the Plücker relation

$$
\begin{aligned}
0= & |-1,0,1, \ldots, N-2| \times\left|0_{l+1}, 1, \ldots, N-2, N-1\right| \\
& -\left|0_{l+1}, 0,1, \ldots, N-2\right| \times|-1,1, \ldots, N-2, N-1| \\
& -|0,1, \ldots, N-2, N-1| \times\left|-1,0_{l+1}, 1, \ldots, N-2\right|,
\end{aligned}
$$

we obtain

$$
\begin{aligned}
0= & \sigma_{n}^{m}(l ; s-1) \times \sigma_{n}^{m}(l+1 ; s)-d_{l} \sigma_{n}^{m}(l+1 ; s-1) \times \partial_{y} \sigma_{n}^{m}(l ; s) \\
& -\sigma_{n}^{m}(l ; s) \times\left[-\left(d_{l} \partial_{y}-1\right) \sigma_{n}^{m}(l+1 ; s-1)\right],
\end{aligned}
$$

which is equivalent to equation (A.7). This completes the proof.

From Proposition A.1 and equation (A.1), we see that $\tau_{n}^{m}(k, l, N ; s)$ satisfies

$$
\begin{gathered}
D_{y} \tau_{n}^{m}(N+1 ; s) \cdot \tau_{n}^{m}(N ; s)=\tau_{n}^{m}(N ; s+1) \tau_{n}^{m}(N+1 ; s-1), \\
D_{y} \tau_{n}^{m}(l+1 ; s) \cdot \tau_{n}^{m}(l ; s+1)=-\frac{1}{d_{l}} \tau_{n}^{m}(l+1 ; s+1) \tau_{n}^{m}(l ; s) .
\end{gathered}
$$


We finally obtain equations (5.26) and (5.27) from equations (A.23) and (A.24), respectively, by imposing the reduction condition (3.28).

\section{REFERENCES}

[1] M. J. Ablowitz, B. Prinari and A. D. Trubatch. Discrete and Continuous Nonlinear Schrödinger Systems. Cambridge University Press, Cambridge, 2004.

[2] L. Bianchi. Sulla trasformazione di Bäcklund per le superficie pseudosferiche. Rend. Lincei 5 (1892), 3-12.

[3] A. Bobenko and U. Pinkall. Discrete surface with constant negative Gaussian curvature and the Hirota equation. J. Differential Geom. 43 (1996), 527-611.

[4] A. I. Bobenko and Y. B. Suris. Discrete Differential Geometry. American Mathematical Society, Providence, RI, 2008.

[5] E. Date, M. Jimbo and T. Miwa. Method for generating discrete soliton equations. I. J. Phys. Soc. Jpn. 51 (1982), 4116-4124.

[6] E. Date, M. Jimbo and T. Miwa. Method for generating discrete soliton equations. II. J. Phys. Soc. Jpn. 51 (1982), 4125-4131.

[7] E. Date, M. Jimbo and T. Miwa. Method for generating discrete soliton equations. III. J. Phys. Soc. Jpn. 52 (1983), 388-393.

[8] E. Date, M. Jimbo and T. Miwa. Method for generating discrete soliton equations. IV. J. Phys. Soc. Jpn. 52 (1983), 761-765.

[9] E. Date, M. Jimbo and T. Miwa. Method for generating discrete soliton equations. V. J. Phys. Soc. Jpn. 52 (1983), 766-771.

[10] A. Doliwa. Geometric discretization of the Toda system. Phys. Lett. A 234 (1997), 187-192.

[11] A. Doliwa and P. M. Santini. Integrable dynamics of a discrete curve and the Ablowitz-Ladik hierarchy. J. Math. Phys. 36 (1995), 1259-1273.

[12] A. Doliwa and P. M. Santini. The integrable dynamic of a discrete curve. Symmetries and Integrability of Difference Equations. Eds. D. Levi, L. Vinet and P. Winternitz. American Mathematical Society, Providence, RI, 1996, pp. 91-102.

[13] A. Doliwa and P. M. Santini. Geometry of discrete curves and lattices and integrable difference equations. Discrete Integrable Geometry and Physics. Eds. A. Bobenko and R. Seiler. Clarendon Press, Oxford. 1999, pp. 139-154.

[14] R. E. Goldstein and D. M. Petrich. The Korteweg-de Vries hierarchy as dynamics of closed curves in the plane. Phys. Rev. Lett. 67 (1991), 3203-3206.

[15] H. Hasimoto. A soliton on a vortex filament. J. Fluid. Mech. 11 (1972), 477-485.

[16] R. Hirota. Nonlinear partial difference equations. I. A difference analogue of the Korteweg-de Vries equation. J. Phys. Soc. Jpn. 43 (1977), 1429-1433.

[17] R. Hirota. Nonlinear partial difference equations. II. Discrete-time Toda equation. J. Phys. Soc. Jpn. 43 (1977), 2074-2078.

[18] R. Hirota. Nonlinear partial difference equations. III. Discrete sine-Gordon equation. J. Phys. Soc. Jpn. 43 (1977), 2079-2086.

[19] R. Hirota. Nonlinear partial difference equations. IV. Bäcklund transformation for the discrete-time Toda equation. J. Phys. Soc. Jpn. 45 (1978), 321-332.

[20] R. Hirota. Nonlinear partial difference equations. V. Nonlinear equations reducible to linear equations. J. Phys. Soc. Jpn. 46 (1979), 312-319.

[21] R. Hirota. Discretization of the potential modified KdV equation. J. Phys. Soc. Jpn. 67 (1998), 2234-2236.

[22] R. Hirota. The Direct Method in Soliton Theory (Cambridge Tracts in Mathematics, 155). Cambridge University Press, Cambridge, 2004.

[23] M. Hisakado, K. Nakayama and M. Wadati. Motion of discrete curves in the plane. J. Phys. Soc. Jpn. 64 (1995), 2390-2393.

[24] T. Hoffmann. Discrete Hashimoto surfaces and a doubly discrete smoke-ring flow. Discrete Differential Geometry (Oberwolfach Seminars, 39). Eds. A. I. Bobenko, P. Schröder, J. M. Sullivan and G. M. Ziegler. Birkhäuser, Basel, 2008, pp. 95-115.

[25] T. Hoffmann. Discrete Differential Geometry of Curves and Surfaces (COE Lecture Notes, 18). Kyushu University, Fukuoka, 2009.

[26] T. Hoffmann and N. Kutz. Discrete curves in $\mathbb{C} P^{1}$ and the Toda lattice. Stud. Appl. Math. 113 (2004), 31-55. 
[27] M. Jimbo and T. Miwa. Solitons and infinite dimensional Lie algebras. Publ. Res. Inst. Math. Sci. 19 (1983), 943-1001.

[28] G. Lamb Jr. Solitons and the motion of helical curves. Phys. Rev. Lett. 37 (1976), 235-237.

[29] K. Maruno, K. Kajiwara and M. Oikawa. Casorati determinant solution for the discrete-time relativistic Toda lattice equation. Phys. Lett. A 241 (1998), 335-343.

[30] K. Maruno and Y. Ohta. Casorati determinant form of dark soliton solutions of the discrete nonlinear Schrödinger equation. J. Phys. Soc. Jpn. 75 (2006), 054002.

[31] N. Matsuura. Discrete KdV and discrete modified KdV equations arising from motions of discrete planar curves. Int. Math. Res. Not. IMRN Advance Access published May 15, 2011, doi:10.1093/imrn/rnr080.

[32] T. Miwa. On Hirota's difference equations. Proc. Jpn. Acad. Ser. A Math. Sci. 58 (1982) 9-12.

[33] F. W. Nijhoff and H. Capel. The discrete KdV equation. Acta Appl. Math. 39 (1995), 133-158.

[34] Y. Ohta, R. Hirota, S. Tsujimoto and T. Imai. Casorati and discrete Gram type determinant representations of solutions to the discrete KP hierarchy. J. Phys. Soc. Jpn. 62 (1993), 1872-1886.

[35] Y. Ohta, K. Kajiwara, J. Matsukidaira and J. Satsuma. Casorati determinant solution for the relativistic Toda lattice equation. J. Math. Phys. 34 (1993), 5190-5204.

[36] U. Pinkall, B. Springborn and S. Weißmann. A new doubly discrete analogue of smoke ring flow and the real time simulation of fluid flow. J. Phys. A: Math. Theor. 40 (2007), 12563-12576.

[37] C. Rogers and W. K. Schief. Bäcklund and Darboux Transformations: Geometry and Modern Applications in Soliton Theory (Cambridge Texts in Applied Mathematics). Cambridge University Press, Cambridge, 2002.

[38] Y. B. Suris. The Problem of Integrable Discretization. Birkhäuser, Basel, 2003.

[39] S. Tsujimoto. On a discrete analogue of the two-dimensional Toda lattice hierarchy. Publ. Res. Inst. Math. Sci. 38 (2002), 113-133.

[40] K. Ueno and K. Takasaki. Toda lattice hierarchy. Group Representations and Systems of Differential Equations (Adv. Stud. Pure Math. 4). Kinokuniya, Tokyo, 1982, pp. 1-95.

[41] M. Wadati. Bäcklund transformation for solutions of the modified Korteweg-de Vries equation. J. Phys. Soc. Jpn. 36 (1974), 1498.

\section{Jun-ichi Inoguchi \\ Department of Mathematical Sciences Yamagata University \\ 1-4-12 Kojirakawa-machi \\ Yamagata 990-8560 \\ Japan}

(E-mail:inoguchi@sci.kj.yamagata-u.ac.jp)

\author{
Kenji Kajiwara \\ Institute of Mathematics for Industry \\ Kyushu University \\ 744 Motooka \\ Fukuoka 819-8581 \\ Japan \\ (E-mail:kaji@imi.kyushu-u.ac.jp)
}

\author{
Yasuhiro Ohta \\ Department of Mathematics \\ Kobe University \\ Rokko \\ Kobe 657-8501 \\ Japan
}

(E-mail:ohta@math.sci.kobe-u.ac.jp)

(E-mail: nozomu@fukuoka-u.ac.jp) 